\title{
miR-7 inhibits glioblastoma growth by simultaneously interfering with the PI3K/ATK and Raf/MEK/ERK pathways
}

\author{
ZHENLIN LIU ${ }^{1}$, ZHONGMIN JIANG $^{2}$, JIANYONG HUANG ${ }^{4}$, SHUQIANG HUANG $^{4}$, \\ YANXIA $\mathrm{LI}^{3}$, SIMIAO YU ${ }^{1}$, SHIZHU YU ${ }^{5-7}$ and XIAOZHI LIU ${ }^{1,4}$ \\ Departments of ${ }^{1}$ Neurosurgery and ${ }^{2}$ Pathology, ${ }^{3}$ The Central Laboratory, The Fifth Central Hospital of Tianjin, \\ Tianjin, P.R. China; ${ }^{4}$ Department of Biomedical Engineering, Duke University, Durham, NC, USA; \\ ${ }^{5}$ Tianjin Neurological Institute, Tianjin Medical University General Hospital; ${ }^{6}$ Key Laboratory of Post-trauma \\ Neuro-repair and Regeneration in Central Nervous System, Ministry of Education; ${ }^{7}$ Tianjin Key Laboratory \\ of Injuries, Variations and Regeneration of Nervous System, Tianjin, P.R. China
}

Received December 29, 2013; Accepted February 14, 2014

DOI: 10.3892/ijo.2014.2322

\begin{abstract}
Epidermal growth factor receptor (EGFR) signaling regulates glioblastoma cell proliferation, survival, migration and invasion and plays a key role in tumor progression. We show that microRNA-7 (miR-7) is a common regulator of the phosphoinositide-3-kinase (PI3K)/ATK and Raf/ mitogen-activated protein kinase kinase (MEK)/extracellular signal-regulated kinase (ERK) pathways, both of which are launched by EGFR through its two direct targets, the transcription factors PI3K and Raf-1, respectively. Enforced expression of miR-7 markedly decreased expression of PI3K, phosphorylated Akt, Raf-1, phosphorylated MEK 1/2, and cyclin D1, as well as slightly reduced expression of EGFR. Forced expression of PI3K or Raf-1 transcripts lacking the 3'-untranslated region (3'-UTR) partially reversed the effects of miR-7 on cell growth inhibition and cell cycle arrest in glioma cells. Additionally, transient expression of miR-7 in glioblastoma cells strongly inhibited in vivo glioblastoma xenograft growth. We conclude that miR-7 is a potential tumor suppressor in glioblastoma that acts by targeting multiple oncogenes related to the downstream pathway of EGFR and may serve as a novel therapeutic target for malignant gliomas.
\end{abstract}

\section{Introduction}

Multiple molecular dysfunctions have been associated with glioblastoma multiforme (GBM) formation and growth (1-3). Among these, EGFR plays a vital role in various aspects of

Correspondence to: Professor Xiaozhi Liu, Department of Neurosurgery, The Fifth Central Hospital of Tianjin, 41 Zhejiang Road, Tanggu, Tianjin 300450, P.R. China

E-mail: 1xz7997@126.com

Key words: microRNA-7, glioma, epidermal growth factor receptor, phosphoinositide-3-kinase malignancy, particularly in tumor cell proliferation, survival and metastasis (4,5). Glioma-associated EGFR mutant forms show constitutive kinase activity that chronically stimulates Ras signaling to drive cell cycle progression and also activates the PI3K/AKT pathway to promote cellular proliferation and migration (6-8).

MicroRNAs modulate protein expression by binding to the 3'UTR of mRNA and promoting RNA degradation, inhibiting mRNA translation, and affecting transcription (9-12). The miR-7 gene is found in most sequenced urbilateria species, and the sequence of its mature miRNA product is perfectly conserved from annelids to humans, indicating strong functional conservation (13-15). Increasing evidence has indicated that miR-7 is a potential tumor suppressor in several human breast and non-small cell lung cancers $(16,17)$. However, thus far, a limited number of target genes have been identified, and the biological function of miR-7 in GBM remains to be further elucidated.

miR-7, which is expressed only in normal brain and pancreatic tissue, demonstrates a high degree of tissue specificity (18) and may be an ideal target for cancer therapy. To further characterize the potential of miR-7 as a target for treating GBM and to clarify its role in the response, we used both in vitro and in vivo systems to investigate the effect of miR-7 suppression in GBM cell lines. We found that overexpression of miR-7 not only suppressed GBM cell proliferation, induced cell apoptosis, and inhibited cell migration in vitro but also reduced tumorigenicity in vivo. Bioinformatics predictions revealed at least four potential binding sites of miR-7 in the 3'-UTR of EGFR, PI3K, and Raf-1. Further research, however, by luciferase assay, confirmed that the PI3K and Raf-1 mRNAs are both direct targets of miR-7, but no clear targeting relationship between EGFR and miR-7 was identified in subsequent experiments. Therefore, we postulate that miR-7 inhibits simultaneously the PI3K/ATK and Raf/MEK/ ERK pathways through its two direct targets, the transcription factors PI3K and Raf-1, which are both located downstream of EGFR. These findings provide a basic rationale for the use of miR-7 in the treatment of malignant brain tumors, such as GBM. 


\section{Materials and methods}

Reagents, animals, and patient tissues. The 2'-O-methyl (2'-O$\mathrm{Me})$ oligonucleotides were chemically synthesized by SBS Genetech (Beijing, China). The 2'-O-Me oligos were composed entirely of $2^{\prime}-\mathrm{O}-\mathrm{Me}$ bases with the following sequences: scrambledsequence:5'-AAGGCAAGCUGACCCUGAAGU-3'; 2'-O-Me-miR-7: 5'-UGGAAGACUAGUGAUUUUGUUGU-3'. The pGL3-WT-EGFR (or PI3K or Raf-1)-3'-UTR-Luc reporter was created by ligation of the polymerase chain reaction (PCR) products of the 3'-UTR of EGFR (or PI3K or Raf-1) into the $X b a \mathrm{I}$ site of the pGL3 control vector (Promega, Madison, WI, USA). The pGL3-MUT-EGFR (or PI3K or Raf-1)-3'-UTR-Luc reporter was generated from pGL3-WT-EGFR (or PI3K or Raf-1)-3'-UTR-Luc reporter by deleting the binding site for miR-7. BALB/c-A nude mice at 6 weeks of age were purchased from the animal center of the Cancer Institute of the Chinese Academy of Medical Sciences. All experimental procedures were carried out according to the regulations and internal biosafety and bioethics guidelines of Tianjin Medical University and the Tianjin Municipal Science and Technology Commission. Tissue specimens of GBM patients were collected after informed consent was received and immediately frozen in liquid nitrogen. The patients or their family member agreed and signed a consent to enroll in the study.

Cell culture and transfection. The human CHG5 glioblastoma cell lines were purchased from Chinese Academy of Sciences. Human glioblastoma cell lines TJ899 and TJ905 were established and characterized in Tianjin Neurological Institute. All cell lines were maintained in Dulbecco's modified Eagle's medium (DMEM) (Invitrogen, Carlsbad, CA, USA) supplemented with $10 \%$ fetal bovine serum (Invitrogen), $100 \mathrm{U} / \mathrm{ml}$ penicillin (Sigma, St. Louis, MO, USA), and $100 \mu \mathrm{g} / \mathrm{ml}$ streptomycin (Sigma), at $37^{\circ} \mathrm{C}$ with $5 \% \mathrm{CO}_{2}$. Oligonucleotides $(50 \mathrm{~nm} / \mathrm{l})$ were transfected into CHG5, TJ899 and TJ905 cells at $70 \%$ confluence using Lipofectamine 2000 (Invitrogen) following the manufacturer's instructions.

MicroRNA array analysis. Total RNA was extracted from GBM cells (CHG5, TJ866 and TJ905) and human normal glial cells using TRIzol reagent (Invitrogen) for miRNA profile examination. The miRNA microarray was obtained from CapitalBio Corp. (Beijing, China). Raw data were normalized to the normal brain cell RNA level and analyzed using the significance analysis of microarrays (SAM) software (version 2.1; http://www-stat.stanford.edu/Btibs/SAM, Stanford University, Stanford, CA, USA).

Real-time reverse transcription ( $R T)$-PCR. Real-time RT-PCR was carried out using the miRNA detection kit (Ambion). Amplification was performed for 40 cycles, each consisting of $95^{\circ} \mathrm{C}$ for $3 \mathrm{~min}, 95^{\circ} \mathrm{C}$ for $15 \mathrm{sec}$, and $60^{\circ} \mathrm{C}$ for $30 \mathrm{sec}$. Both the RT and PCR primers were purchased from Ambion, and 5S RNA was used for normalization.

Cell viability assay. Cell viability was determined using the 3-(4,5-dimethylthiazol-2-yl)-2,5-diphenyl tetrazolium bromide (MTT, Sigma) assay according to the manufacturer's instructions.
Apoptosis assays. Cells were plated in 12-well plates and transfected with oligonucleotides. The apoptosis ratio was analyzed $24 \mathrm{~h}$ after transfection using an Annexin V FITC apoptosis detection kit (BD Biosciences, Franklin Lakes, NJ, USA) according to the manufacturer's instructions.

Cell cycle analysis. Cells were collected by trypsinization, washed in PBS, and fixed in $70 \%$ ethanol for $30 \mathrm{~min}$ at $4^{\circ} \mathrm{C}$. Next, cells were incubated with the DNA-binding dye PI $(50 \mu \mathrm{g} / \mathrm{ml})$ and RNase $(1.0 \mathrm{mg} / \mathrm{ml})$ for $30 \mathrm{~min}$ at $37^{\circ} \mathrm{C}$ in the dark. Finally, cells were washed, and red fluorescence was analyzed by a FACSCalibur flow cytometer (BD Biosciences, San Jose, CA, USA) using a peak fluorescence gate to discriminate aggregates.

Cell invasion assessment. Cell invasion abilities were examined using 6-well Transwell chambers and a reconstituted extracellular matrix membrane (BD Biosciences, San Jose, CA, USA). After allowing Matrigel polymerization, the cells treated with miR-7 mimic or scrambled sequences were added to the upper chambers. The migrated cells were counted microscopically $(x 400)$ in five fields per filter.

Colony formation assay. Colony formation was evaluated as described previously (19). Briefly, 250-300 cells were placed in a 6-well plate and maintained in DMEM containing $10 \%$ FBS for 7-14 days. Colonies were fixed with methanol and stained with $0.1 \%$ crystal violet in $20 \%$ methanol for $15 \mathrm{~min}$, and representative colonies were photographed.

Luciferase assays. For reporter assays, TJ899 cells were transfected with miR-7 mimic or scrambled sequences for $48 \mathrm{~h}$ and subsequently cultured in 96-well plates and transfected with pGL3-WT-EGFR (or PI3K or Raf-1)-3'-UTR-Luc, or pGL3-MUT-EGFR (or PI3K or Raf-1)-3'-UTR-Luc. After $48 \mathrm{~h}$, luciferase assays were performed using the Luciferase System kit, and luminescence was measured on a Promega GloMax 20/20 luminometer and normalized as previously described (20).

Western blotting. Western blotting was performed as previously described (21). Proteins were incubated with primary antibodies against PI3K, Raf-1, and cyclin D1 (Santa Cruz Biotechnology, Santa Cruz, CA, USA), as well as EGFR, p-AKT, and p-MEK1/2 (Zhongshan Bio Corp., Beijing, China) followed by incubation with an HRP-conjugated secondary antibody (Zymed, San Francisco, CA, USA). Proteins were detected using a SuperSignal protein detection kit (Pierce, Rockford, IL, USA).

Xenograft tumor assay. Thirty-two mice were randomly divided into 4 groups. Glioma subcutaneous model was established as previously described (22). A mixture of $10 \mu \mathrm{l}$ of Lipofectamine 2000 and $10 \mu \mathrm{g}$ of oligonucleotides was injected into the xenograft tumor model in a multi-site injection manner. Mice in the control group received only $10 \mu 1$ of Lipofectamine 2000. In the removal group, the agents were removed after 10 days of the treatment. Tumor growth was monitored by caliper measurement every 3 days for 25 days. Tumor volume $(\mathrm{V})$ was calculated as 


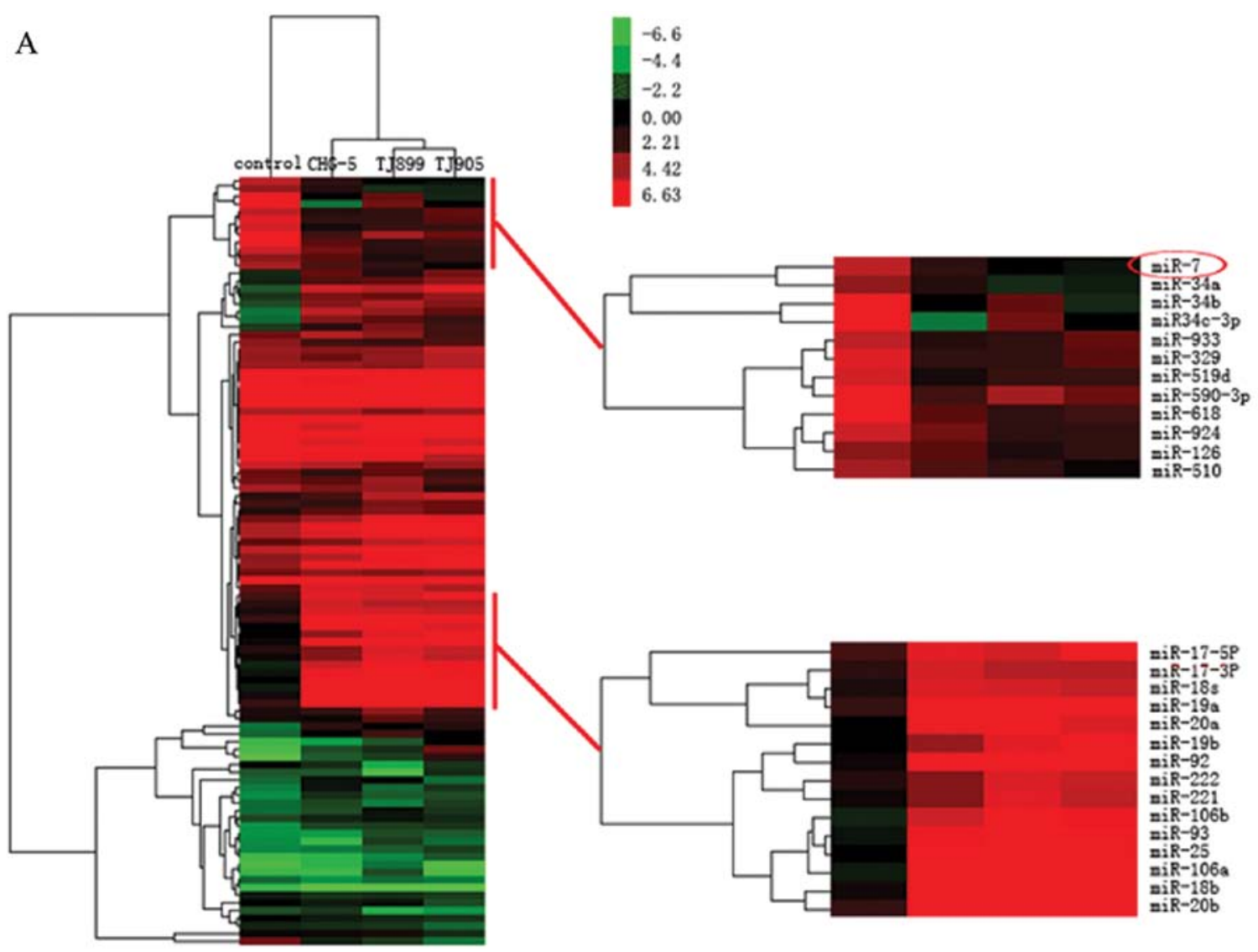

B

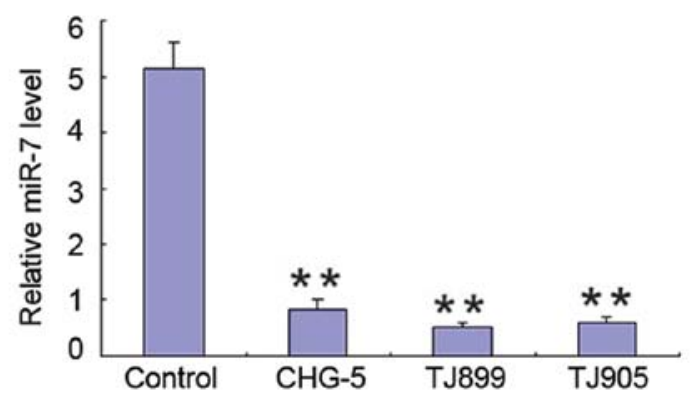

Figure 1. miR-7 is expressed at a low level in GBM cells. (A) Cluster analysis of the miRNA expression profiles of three human GBM cell lines. (B) Real-time RT-PCR indicated low miR-7 expression in GBM cell lines. U6 snRNA was used as a loading control.

follows: $\mathrm{V}=\mathrm{L} \times \mathrm{W}^{2} \times 0.5$; L, length; $\mathrm{W}$, width. Tumor weight was detected at the end of the study.

In situ hybridization assay. Using a locked nucleic acid (LNA)modified antisense oligonucleotide probe, in situ hybridization was performed using the In Situ hybridization kit (Wuhan Boster Biological Technology, Ltd., Wuhan, China). After sections were prehybridized for $2 \mathrm{~h}$ in hybridization liquid, they were incubated with $20 \mu \mathrm{l}$ of LNA-miR-7 hybridization solution at $42^{\circ} \mathrm{C}$ for $16 \mathrm{~h}$, and $\mathrm{Cy} 3$-avidin was used to label miR-7 at a concentration of $0.5 \mathrm{mg} / \mathrm{ml}$.

In situ terminal deoxynucleotidyl transferase dUTP nickend labeling (TUNEL) analysis. Apoptotic cell death was examined by TUNEL analysis using an In Situ Cell Death kit (Roche Carolina Inc., Florence, SC, USA) according to the manufacturer's instructions.
Immunohistochemistry. The Ki67, EGFR, PI3K, p-AKT-2, Raf-1, p-MEK1/2 and cyclin D1 protein levels were determined by immunohistochemistry as previously described (22).

Statistical analysis. Results were analyzed using the SPSS software version 11.0. Data are presented as the mean \pm standard deviation (SD). A P-value of $<0.05$ was taken to indicate statistical significance.

\section{Results}

miR-7 expression was detected in normal brain tissue and at low levels in glioblastoma. Previous studies have reported that miR-7 expression showed high tissue specificity and was expressed in only normal brain tissue, normal pancreatic tissue, and pancreatic tumor tissue. In glioma cell lines, our analysis showed that expression of 7 of the 118 human 

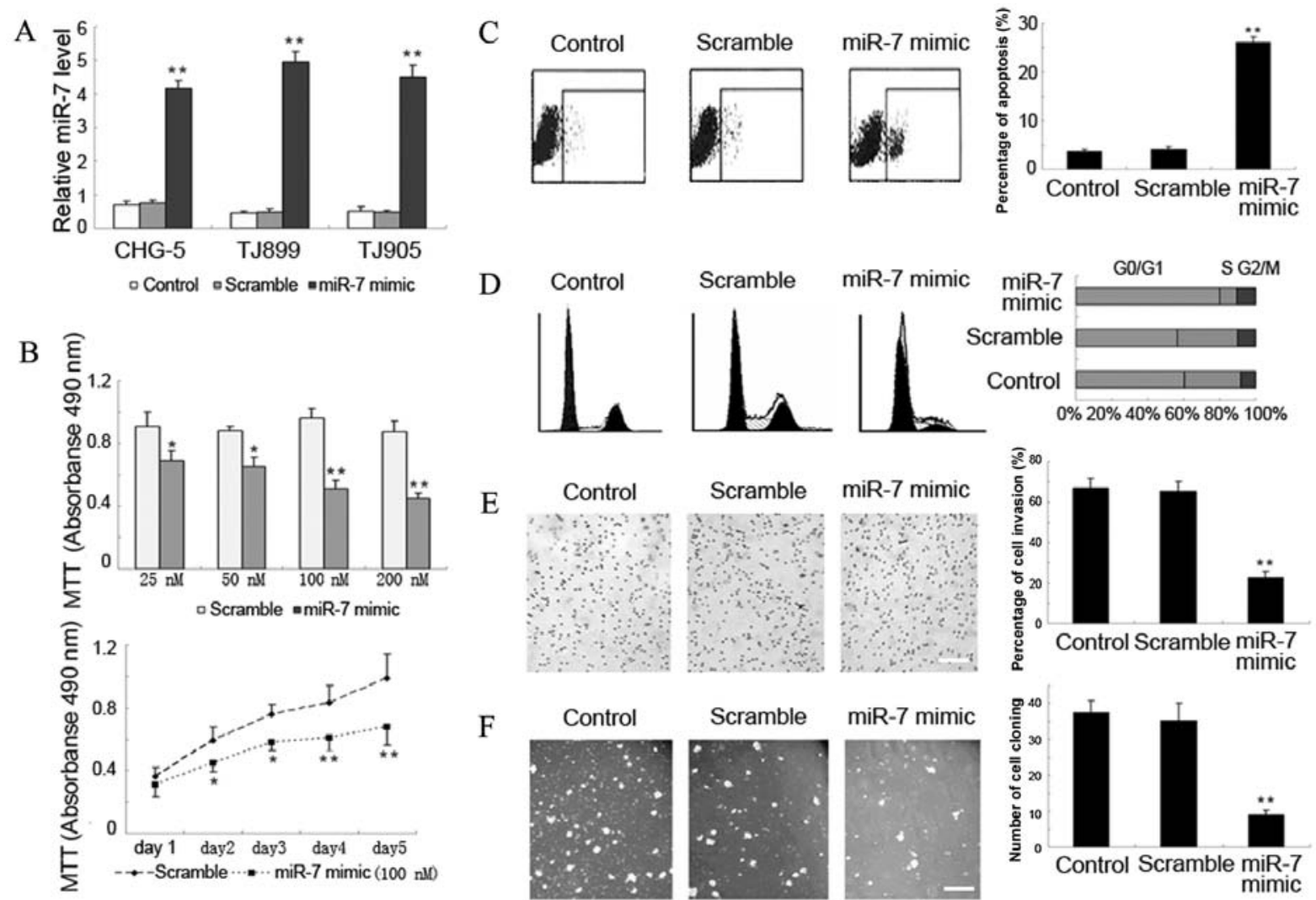

Figure 2. The effect of miR-7 restoration on human GBM cell lines. (A) After transient transfection of miR-7 mimics for $72 \mathrm{~h}$, all three cell lines exhibited at least a 5-fold increased miR-7 expression. (B) The dose- and time-dependent effect of miR-7 mimics on TJ899 cell viability. (C) Annexin V staining showed that TJ899 cells transfected with miR-7 mimics displayed significantly greater apoptosis than the control and scramble groups. (D) Cell cycle profiles after PI staining. miR-7 restoration induced G1 arrest in TJ899 GBM cells. (E) Cell invasiveness was detected by Transwell invasion assay. TJ899 cells were transfected with $100 \mathrm{nM}$ of miR-7 mimics or $100 \mathrm{nM}$ of scrambled sequences. (F) Colony formation in the presence of miR-7 mimics or scrambled sequences. The graph shows three independent experiments. Data represent the means \pm SD. Significant differences compared with the controls are shown as ${ }^{*} \mathrm{P}<0.05 ;{ }^{* *} \mathrm{P}<0.01$. (E and F) Original magnification, $\mathrm{x} 100$.

miRNAs (5.93\%) was more than 2-fold lower, and that of 11 of $118(9.32 \%) 2$-fold higher relative to levels in normal tissue (Fig. 1A). miR-7 showed the most significant decrease relative to normal tissue (6.4-fold), particularly in the TJ899 GBM cell line (7.9-fold). The miR-7 expression data from the microarray analysis were confirmed using quantitative RT-PCR (Fig. 1B).

miR-7 inhibits GBM malignancy. We assessed the effects of miR-7 on GBM malignancy variables, including cell proliferation, cell cycle, cell death, and cell invasion in three GBM cell lines from Chinese patients. We first performed in vitro gain-of-function analyses by introducing miR-7 mimics into CHG-5, TJ899 and TJ905 cells. The high expression levels of miR-7 in all the three cell lines were found to be at least 5 times that of the control or scramble group $72 \mathrm{~h}$ after the miR-7 mimics were transiently transfected into the cells (Fig. 2A). Ectogenic miR-7 may significantly inhibit cell proliferation in a dose- and time-dependent manner (Fig. 2B), induce apoptosis (Fig. 2C) and cell cycle arrest (Fig. 2D), and suppress cell invasiveness (Fig. 2E) as well as colony formation (Fig. 2F). On the third day of the miR-7 mimic transfection at doses of $25,50,100$ or $200 \mathrm{nM}$, the cell proliferation decreased gradually with the increase in dose. After 2 days of miR-7 mimic transfection at $100 \mathrm{nM}$ dose, miR-7 restoration inhibited cell proliferation significantly. The miR-7 expression induced cell death from $3.6 \pm 0.6 \%$ to $21.4 \pm 2.1 \%$ in CHG-5 cells $(\mathrm{n}=3$; $\mathrm{P}<0.05)$, from $3.7 \pm 0.4 \%$ to $26.1 \pm 1.1 \%$ in TJ899 cells $(\mathrm{n}=3$; $\mathrm{P}<0.05$ ), and from $1.8 \pm 0.2 \%$ to $16.4 \pm 0.5 \%$ in TJ905 cells $(\mathrm{n}=3$; $\mathrm{P}<0.05)$. MiR-7 also significantly induced cell cycle arrest and increased the G0-G1 fraction in the three Chinese cell lines. Moreover, miR-7 restoration also resulted in a remarkable increase of cell invasiveness from $73.9 \pm 4.0 \%$ to $91.7 \pm 2.1 \%$ in CHG-5 cells $(n=3 ; P<0.05)$, from $67.1 \pm 4.4 \%$ to $22.5 \pm 3.4 \%$ in TJ899 cells $(n=3 ; P<0.05)$, and from $68.8 \pm 1.1 \%$ to $86.4 \pm 1.4 \%$ in TJ905 cells $(n=3 ; P<0.05)$. Ectogenic miR-7 significantly promoted colony formation from $36.9 \pm 2.8 \%$ to $11.7 \pm 2.2 \%$ in CHG-5 cells $(n=3 ; P<0.05)$, from $37.4 \pm 3.4 \%$ to $8.9 \pm 1.4 \%$ in TJ899 cells $(\mathrm{n}=3 ; \mathrm{P}<0.05)$, and from $28.8 \pm 1.7 \%$ to $11.4 \pm 1.2 \%$ in TJ905 cells $(n=3 ; P<0.05)$.

miR-7 inhibits the expression of PI3K and Raf- 1 and binds to their 3'-UTR. The TargetScan and Pictar target prediction databases found the 3'-UTR of EGFR $\left(\mathrm{P}_{\mathrm{CT}}=0.61\right)$, PI3K $\left(\mathrm{P}_{\mathrm{CT}}=0.74\right)$, and Raf- $1\left(\mathrm{P}_{\mathrm{CT}}=0.97\right)$ to contain the highly conserved putative miR-7 binding sites (Fig. 3A). To determine whether miR-7 could directly inhibit EGFR, PI3K, and Raf-1 protein expression by binding to their 3'-UTR, we created pGL3-WT-EGFR (or PI3K or Raf-1)-3'-UTR and pGL3-MUT- EGFR (or PI3K or Raf-1)-3'-UTR plasmids. Reporter assays revealed that miR-7 restoration led to a marked decrease in the luciferase activity of the pGL3-WT-PI3K (or Raf-1)-3'-UTR plasmid, but did 


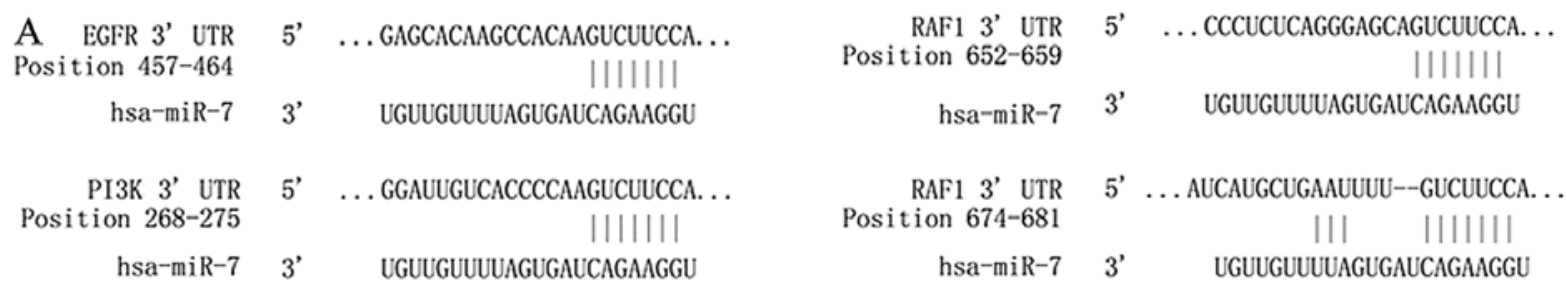

B

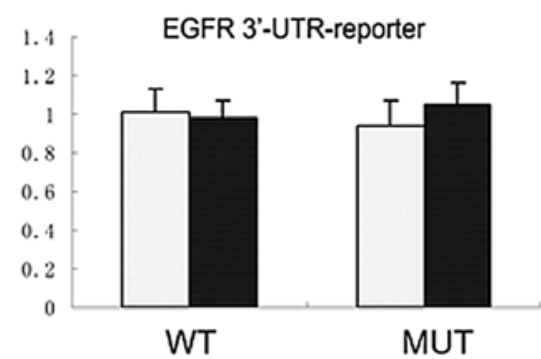

PI3K 3'-UTR-reporter

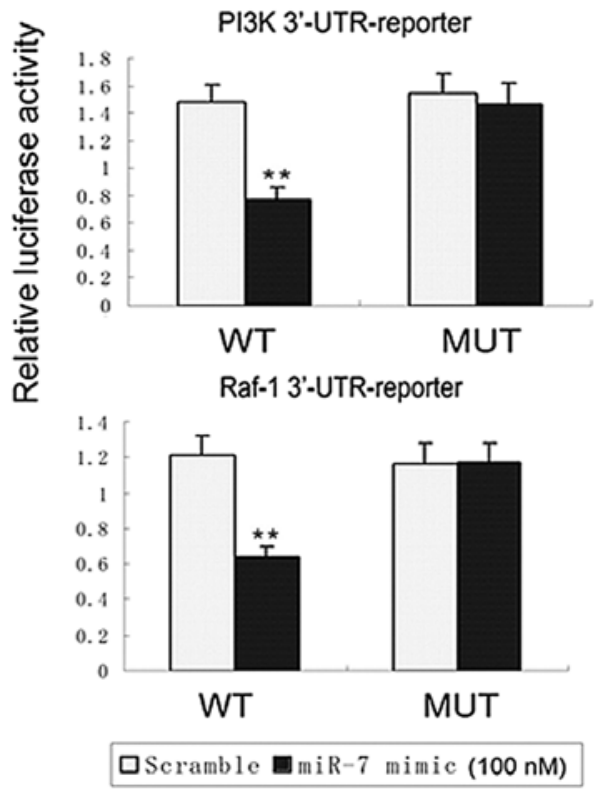

$\mathrm{C}$
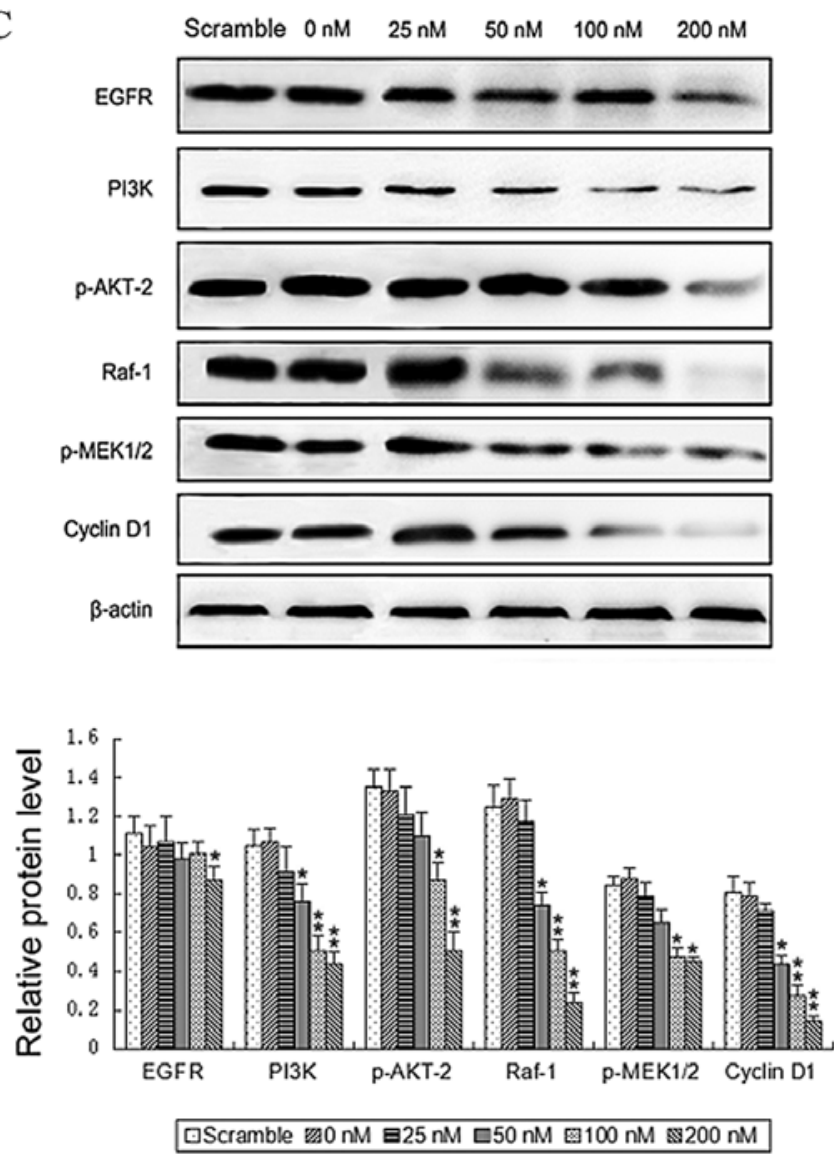

Figure 3. miR-7 inhibits the expression of PI3K and Raf-1 and binds to their 3'-UTR in human GBM cells. (A) The predicted seed matching between miR-7 and the oncogenes' 3'-UTR sequences. (B) Luciferase activity. TJ899 cells were cotransfected with pGL3-WT-EGFR (or PI3K or Raf-1)-3'-UTR and pGL3MUT-EGFR (or PI3K or Raf-1)-3'-UTR plasmids (indicated as WT or MUT on the x-axis), and luciferase activity was assayed $48 \mathrm{~h}$ after transfection. miR-7 expression downregulated the luciferase activities of PI3K and Raf-1, but not that of EGFR, in GBM cells. (C) TJ899 cells were transfected with miR-7 mimics at various doses, and the related protein levels were detected by western blotting. $\beta$-actin protein was used as the endogenous normalizer. The graph is representative of three independent experiments. Data represent the means $\pm \mathrm{SD}$. Significant differences compared with the controls: ${ }^{*} \mathrm{P}<0.05$ and ${ }^{* *} \mathrm{P}<0.01$.

not affect the luciferase activity of the pGL3-MUT-PI3K (or Raf-1)-3'-UTR plasmid. By contrast, there was no statistically significant difference between the pGL3-WT-EGFR-3'-UTR and pGL3-MUT-EGFR-3'-UTR plasmids in terms of luciferase activity (Fig. 3B).

Furthermore, we assessed the effects of miR-7 mimic transfection on the protein levels of all the above oncogenes and several accompanying downstream genes (23-30). Western blotting showed that expression of PI3K and Raf-1 was downregulated in TJ899 cells in a dose-dependent manner after 3 days of miR-7 mimic transfection. EGFR, however, showed only slightly and non-dose-dependently reduced expression. Moreover, both phosphorylated AKT2 (p-AKT2) and p-MEK1/2 levels were downregulated, and the expression of cyclin D1 was decreased (Fig. 3C and D), compared with that in cells treated with scrambled oligonucleotide or vector alone (control group) (data not shown).

Recovery of PI3K and Raf-1 expression overrides the effect of $m i R-7$ in GBM. To further assess the regulatory role of miR-7 in the PI3K/ATK and Raf/MEK/ERK pathways, we transfected PI3K lacking the 3'-UTR and Raf-1 lacking the 3'-UTR into TJ899 cells. Two days later, the expression of PI3K recovered most of the functions abolished by miR-7 mimic transfection in TJ899 cells, including tumor cell proliferation, inhibition of apoptosis, and cell invasion (Fig. 4A-E). However, only slight recovery of the cell cycle was observed, as indicated by a small shortening of the G0/G1 phase and extension of the $\mathrm{S}$ phase, as well as slightly increased cyclin D1 expression (Figs. 4C, 5A and B). The expression of Raf-1 
A

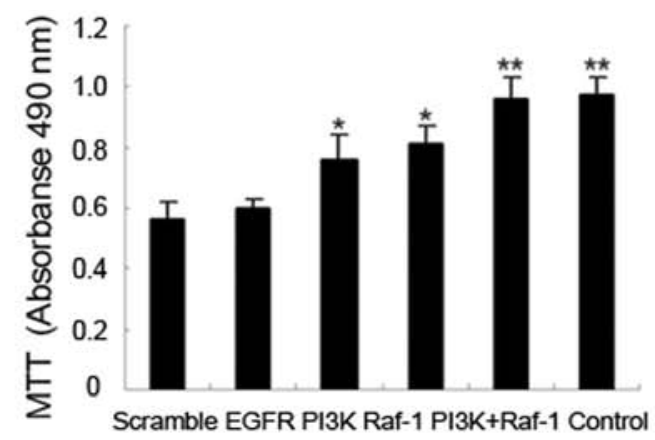

C

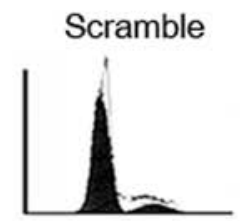

Raf-1
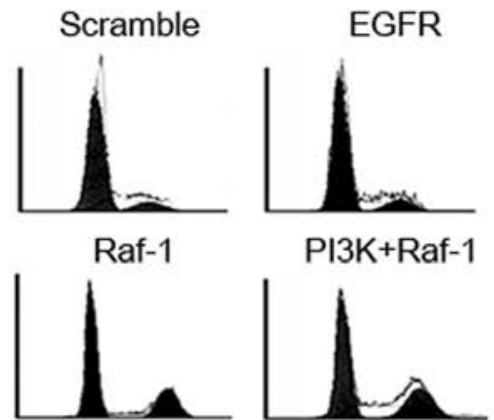

PI3K+Raf-1

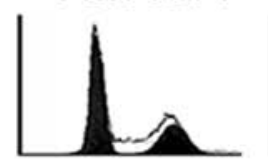

D

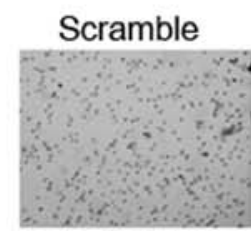

Raf-1
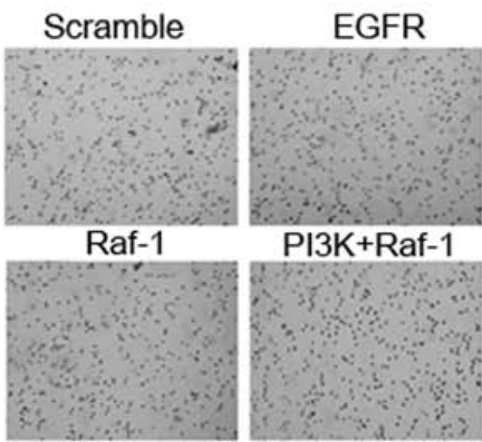

E

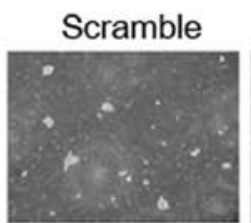

Raf-1

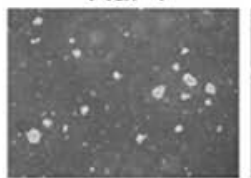

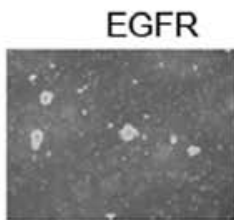

PI3K+Raf-1

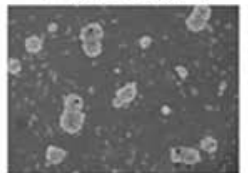

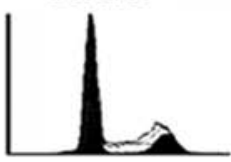

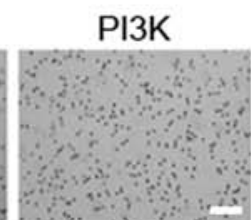

Control

PI3K
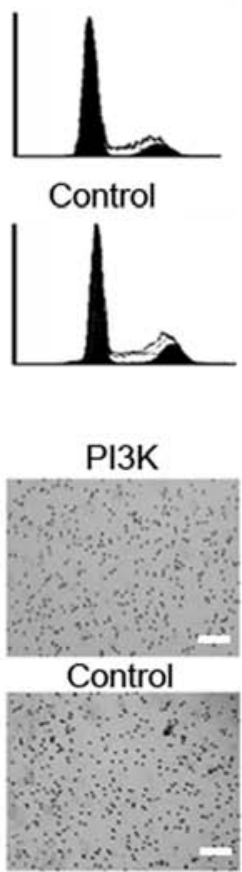

PI3K

Control

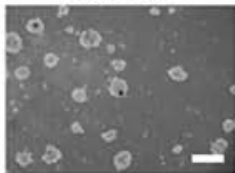

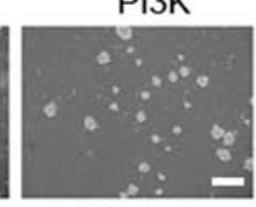

B

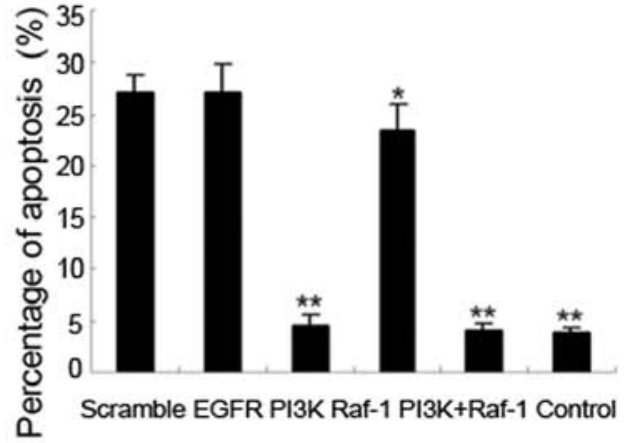

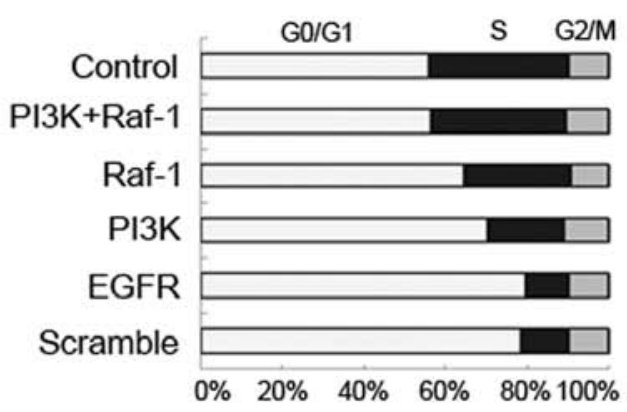
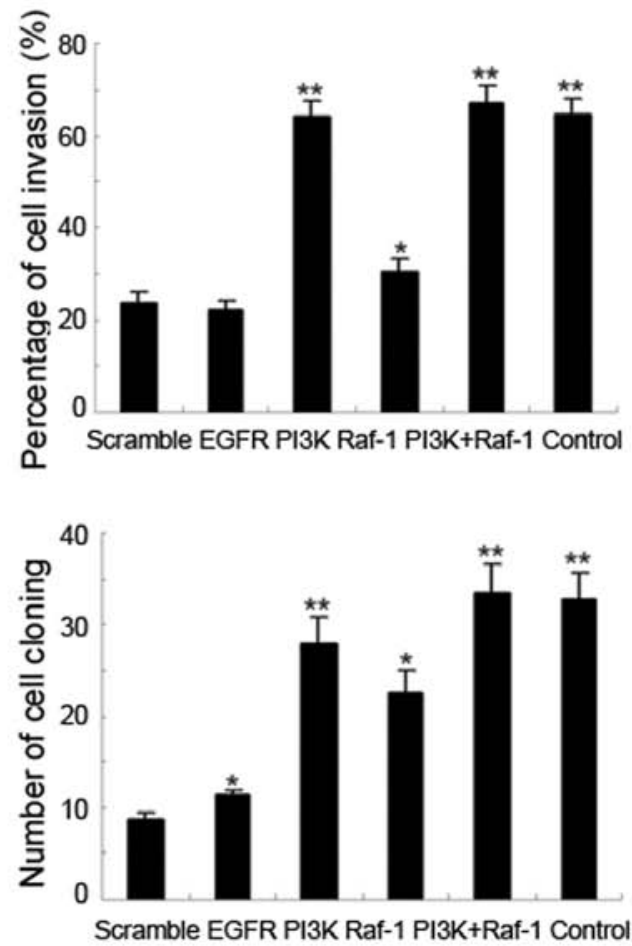

Figure 4. Recovery of PI3K and Raf-1 expression can override miR-7 function in TJ899 cells. TJ899 cells were transfected with pcDNA-EGFR (or PI3K or Raf-1) (not including the 3'-UTR) on the third day after miR-7 mimic transfection at a $100 \mathrm{nM}$ dose. Two days later, tumor cell proliferation, apoptosis, cell cycle changes and invasion were analyzed. PI3K expression recovered most functions abolished by miR-7 mimic transfection in TJ899 cells, including tumor cell proliferation, inhibition of apoptosis and cell invasion; however, only slight recovery of cell cycle progression was observed. (A) TJ899 cell viability by MTT assay. (B) The percentage of apoptotic cells was detected by Annexin V staining. (C) Changes in the cell cycle were detected by flow cytometry. (D) Cell invasiveness was detected by Transwell invasion assay. (E) Colony formation was evaluated using soft agar colony formation assays. The graph is representative of three independent experiments. Data represent the means \pm SD. Significant differences compared with the controls: ${ }^{*} \mathrm{P}<0.05 ;{ }^{* *} \mathrm{P}<0.01$. (D and E) Original magnification, $\mathrm{x} 100$.

largely abrogated the effects of miR-7 on the cell cycle of TJ899 cells, with an obvious shortening of the G0/G1 phase and prominent extension of the $\mathrm{S}$ phase, as well as dramatically increased cyclin D1 expression (Figs. 4C, 5A and B).
Surprisingly, when PI3K lacking the 3'-UTR and Raf-1 lacking the 3'-UTR were transfected simultaneously into TJ899 cells, the tumor cells were almost completely restored to their original state, with high proliferative activity, obvious 

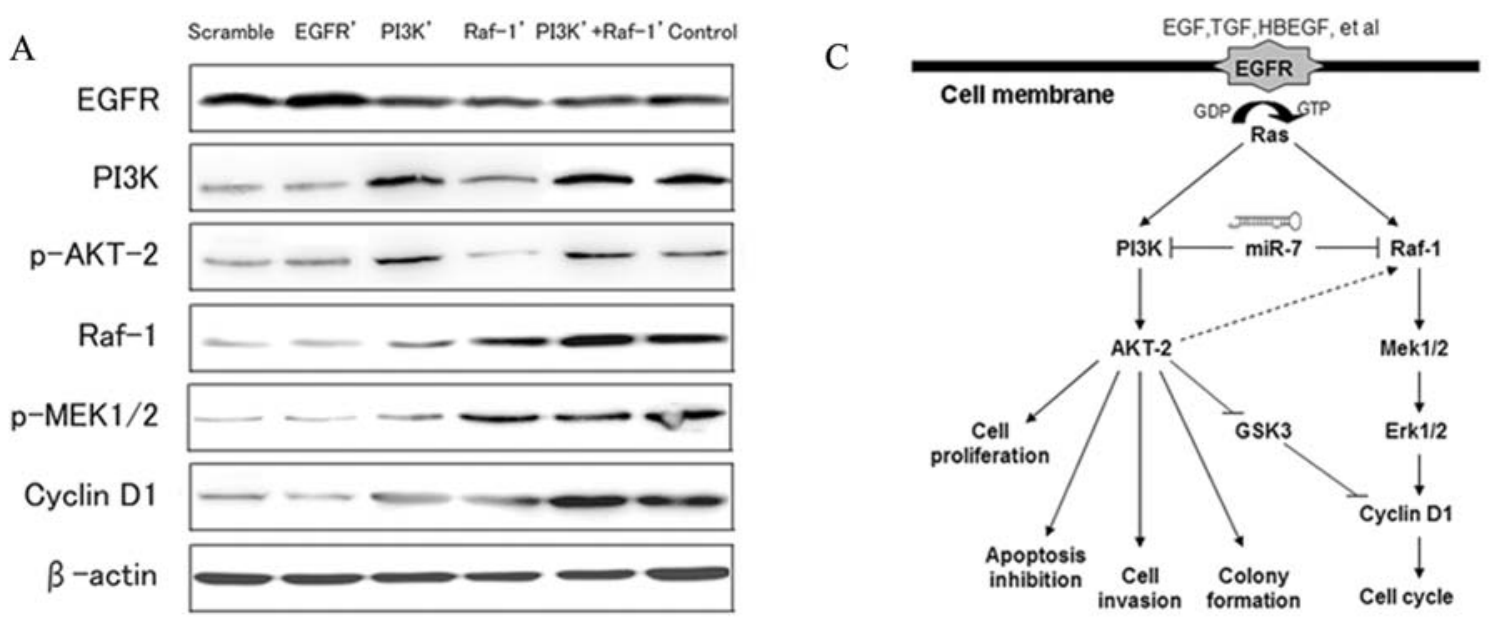

B

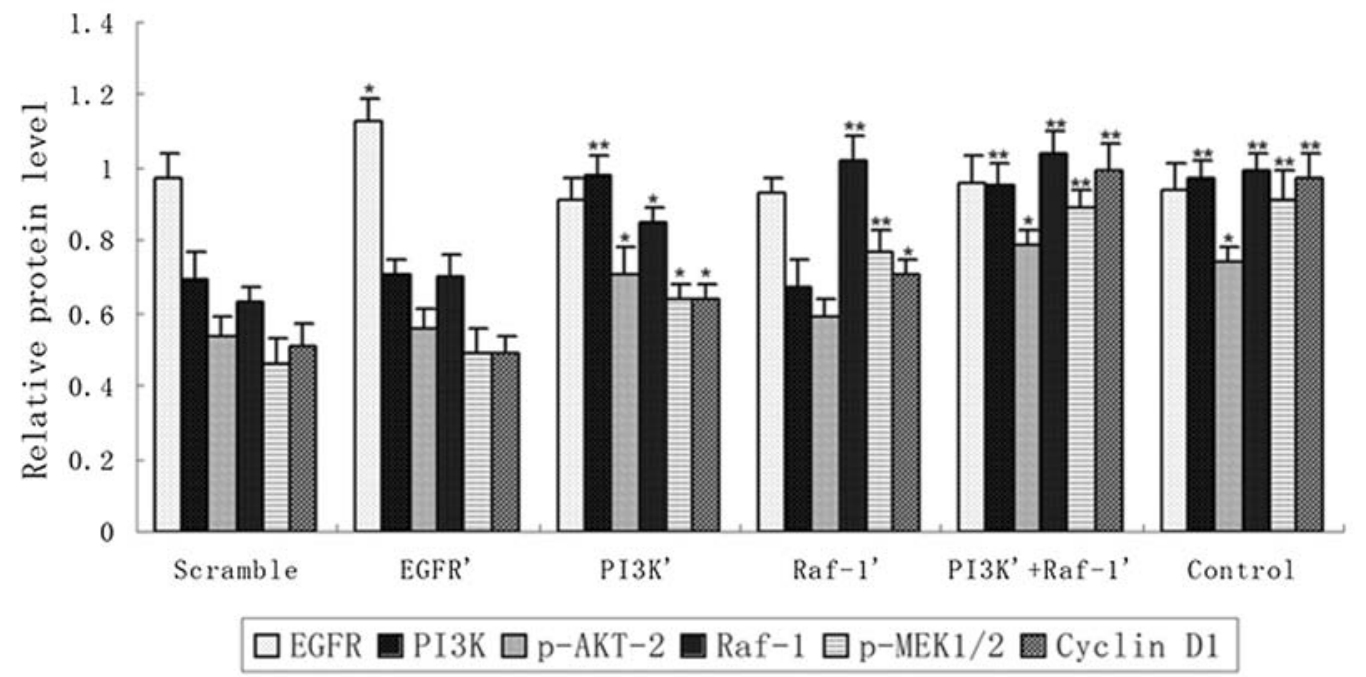

Figure 5. The effects of EGFR, PI3K, and Raf-1 expression (not including the 3'-UTR) on several related proteins in human GBM TJ899 cells. (A and B) The expression levels of 6 related proteins were measured by western blotting. $\beta$-actin protein was regarded as the endogenous normalizer. (C) Proposed regulatory mechanism. The graph represents three independent experiments. Data represent the means \pm SD. Significant differences compared with the controls: ${ }^{*}<0.05$; *** $\mathrm{P}<0.01$

apoptosis inhibition, high invasiveness and rapid cell cycle progression (Fig. 4A-E). Equally surprisingly, transfection of EGFR lacking the 3'-UTR into TJ899 cells had little effect (Fig. 4A-E). These findings further indicated that miR-7 directly modulates PI3K and Raf-1, but not EGFR, expression by binding to the mRNA 3'-UTR.

To clarify the regulatory mechanism, we determined the effect of EGFR, PI3K, and Raf-1 (not including the 3'-UTR) expression on several proteins involved in the PI3K/ATK and Raf/MEK/ERK pathways in human GBM TJ899 cells. Forced PI3K expression significantly increased the level of p-AKT-2 and slightly increased the level of cyclin D1; additionally, Raf-1 expression significantly increased the level of p-MEK1/2 and slightly increased the level of cyclin D1. Of note, forced PI3K expression also slightly increased Raf-1 and p-MEK1/2 protein expression, but forced Raf-1 expression had no effect on PI3K and p-AKT-2 expression levels. We also found that EGFR expression had little impact on the expression levels of downstream proteins, including PI3K, p-AKT-2, Raf-1, p-MEK1/2 and cyclin D1, indicating that miR-7 plays a key role in silencing the downstream pathways of EGFR (Fig. 5A-C).

miR-7 inhibits glioblastoma xenograft growth. Since miR-7 plays an important role in GBM tumor progression, we further examined the effects of miR-7 on tumor growth in vivo. Compared with tumors that continued growing in both the scramble and control groups, the miR-7 group demonstrated a significant reduction in both tumor volume and weight (Fig. 6A-C) $(\mathrm{P}<0.05)$. In another group (the removal group), the tumors started to regrow 10 days after cessation of treatment (Fig. 6A-C). TUNEL assay of xenograft tumors performed 25 days after treatment revealed markedly greater apoptosis in the miR-7 group compared with the scramble and control groups $\left(\chi^{2}=21.74 ; \mathrm{P}<0.01\right.$; Fig. 6D). Ki-67 staining showed that miR-7-treated tumors had a lower proliferation index than control tumors $\left(\chi^{2}=17.35 ; \mathrm{P}<0.05\right.$; Fig. 6E). Additionally, immunohistochemical analysis revealed a marked decrease in the PI3K, Raf-1, and cyclin D1 protein levels in the miR-7treated group $(\mathrm{P}<0.05$; Fig. $6 \mathrm{E})$. These findings confirm that 
A

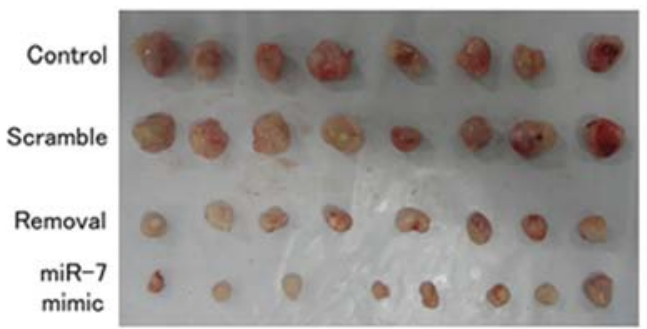

$\mathrm{B}$

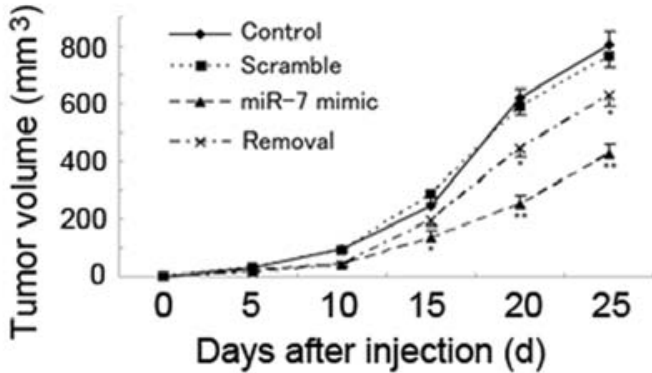

$\mathrm{C}$

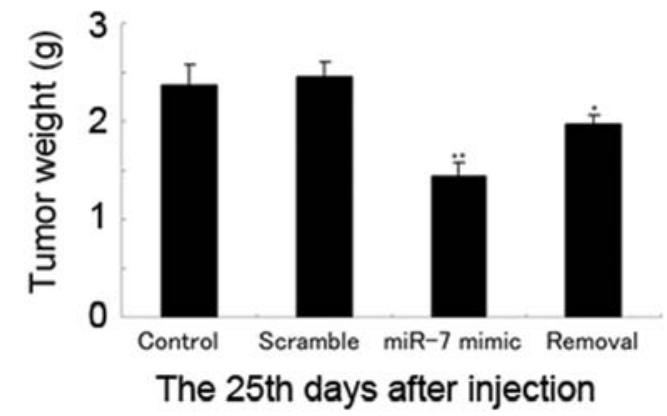

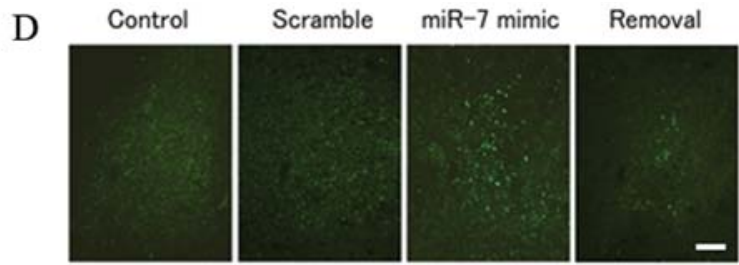

$\mathrm{E}$

Ki67
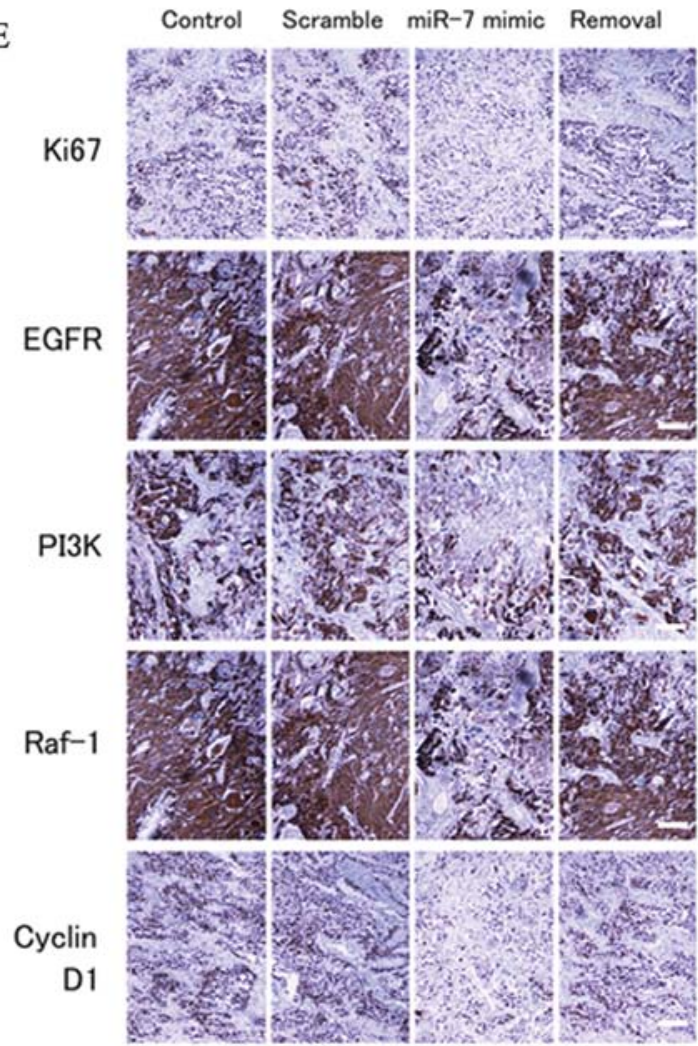

Figure 6. miR-7 inhibits glioblastoma xenograft growth. (A) Tumors were dissected from the subcutaneous region of nude mice. (B) Tumor volume was monitored over 25 days. (C) Tumor weight was determined at the end of the study. (D) TUNEL assay of the xenograft tumor sections revealed that miR-7 induced apoptosis. (E) Ki67, EGFR, PI3K, Raf-1 and cyclin D1 expression levels in xenograft tumor sections were detected by immunohistochemistry. The graph represents three independent experiments. Data represent the means $\pm \mathrm{SD}$. Significant differences compared with the controls: ${ }^{*} \mathrm{P}<0.05 ;{ }^{* *} \mathrm{P}<0.01$. (D and E) Original magnification, x100.

miR-7 targets PI3K and Raf-1 and so could be used for treatment of GBM.

\section{Discussion}

GBM is a leading cause of cancer death involving the central nervous system (31). Unfortunately, the underlying molecular mechanisms of unlimited proliferation and progressive local invasion remain obscure. Previous work has demonstrated that GBM frequently harbors mutations that activate EGFR and launch downstream signaling pathways, including the PI3K/ATK and Ras/Raf/MEK/ERK pathways $(6,8,32)$. However, EGFR-Ras or PI3K mutations alone are not sufficient to transform glial cells; instead, multiple mutations that coactivate the EGFR-Ras and PI3K/Akt pathways are necessary to induce glioma (33). Recently, much attention has focused on miRNAs, a newly discovered family of genes encoding small RNA molecules that bind through partial sequence homology to the 3'-UTRs of target genes, which play key roles in the regulation of gene expression and the protein network (34-38).
In the present study, we reviewed the relevant literature and found that miR-7 demonstrated high tissue specificity, being expressed in only normal brain tissue and pancreatic tissue (18). We hypothesized that miR-7 may have important research value in brain diseases. To identify the mechanism by which miR-7 regulates GBM malignancy, we identified the 3'-UTR of EGFR, PI3K, and Raf-1 to contain highly conserved putative miR-7 binding sites by bioinformatics analysis. However, the expression of PI3K and Raf-1, but not EGFR, were regulated by miR-7 as determined by luciferase activity reporter and western blot assays. We also investigated p-Akt-2, p-MEK1/2 and cyclin D1 levels. Glioma-associated EGFR mutant forms showed constitutive kinase activity that chronically stimulates Ras/Raf/MEK/ERK signaling to drive cell proliferation and cell cycle progression; this is mediated mainly by cyclin D.

Other common genetic lesions include activating mutations in PIK3CA, which encodes the p110a catalytic subunit of PI3K. AKT, a major PI3K effector, regulates the cell cycle and cell proliferation through its direct action on the cyclindependent kinase inhibitors p21 and p27, and its indirect effect on the levels of cyclin D1 and p53, as well as cell survival, 
through direct inhibition of pro-apoptotic signals such as Bad and the Forkhead family of transcription factors $(39,40)$. Our data show that miR-7 expression reduces AKT2 and MEK1/2 phosphorylation levels, as well as cyclin D1 expression. Furthermore, recovery of the PI3K or Raf-1 expression level by transfection of pcDNA-PI3K or pcDNA-Raf-1 (not including the 3'-UTR), respectively, partially abrogated the inhibition by miR-7 of the malignant progression of TJ899 cells. When PI3K and Raf-1, both lacking the 3'-UTR, were transfected simultaneously, the TJ899 tumor cells were almost completely restored to their original state. However, transfection of EGFR lacking the 3'-UTR had little effect on TJ899 cells. We also found that forced PI3K expression slightly increased Raf-1 and $\mathrm{p}-\mathrm{MEK} 1 / 2$ protein levels, but forced Raf-1 expression had no effect on PI3K and p-AKT-2 levels. Therefore, we hypothesized a potential regulatory mechanism between PI3K and Raf-1 or MEK1/2. Taken together, these results revealed that miR-7 is a common regulator of the PI3K/ATK and Ras/Raf/ MEK/ERK pathways, an effect mediated by its targeting of the transcription factors PI3K and Raf-1, but not EGFR. The end result is inhibition of the malignant transformation of GBM cells.

Kefas and others have shown quite convincingly that EGFR is targeted by miR-7 (41). However, our data are not contradictory with their findings, for Kefas has also reported that transfection with the pri-miR-7 vector did not result in decreased EGFR protein in U87MG, and furthermore that there might have a processing defect in generating pre-miR-7 from pri-miR-7 (41). Therefore, there may be a case that the single stranded miR-7 mimic cannot been processed into the RISC complex to target EGFR. However, there are also positive facts that the single stranded miR-7 mimic can effectively silence the two other target genes PI3K and Raf-1, which are both located downstream of EGFR. Moreover, IRS2 has been previously shown to be a target of miR-7 and lies upstream of Akt signaling (41). Therefore, there may be a complex regulatory network involving miR-7, and PI3K/ATK and Ras/Raf/ MEK/ERK pathways that are both launched by EGFR. Taken together, our data provide evidence that miR-7 may represent a key therapeutic target for glioblastoma.

\section{Acknowledgements}

This study was supported by the China National Natural Scientific Fund (81000901), Tianjin Health Bureau Science and Technology Projects (10KG210, 2011KZ24 and 2011KZ26), Tianjin Binhai New Area Health Bureau Science and Technology Projects (2011BHKZ004, 2012BHKZ001 and 2012BHKZ003), and Tianjin Binhai New Area Science and Technology Projects (2012MS05-04).

\section{References}

1. Raizer JJ, Grimm S, Chamberlain MC, et al: A phase 2 trial of single-agent bevacizumab given in an every-3-week schedule for patients with recurrent high-grade gliomas. Cancer 116: 5297-5305, 2010

2. Bonavia R, Inda MM, Cavenee WK and Furnari FB: Heterogeneity maintenance in glioblastoma: a social network. Cancer Res 71: 4055-4060, 2011.

3. Masica DL and Karchin R: Correlation of somatic mutation and expression identifies genes important in human glioblastoma progression and survival. Cancer Res 71: 4550-4561, 2011.
4. Yang RY, Yang KS, Pike LJ and Marshall GR: Targeting the dimerization of epidermal growth factor receptors with smallmolecule inhibitors. Chem Biol Drug Des 76: 1-9, 2010.

5. Tabunoki H, Saito N, Suwanborirux K, et al: Molecular network profiling of U373MG human glioblastoma cells following induction of apoptosis by novel marine-derived anti-cancer 1,2,3,4-tetrahydroisoquinoline alkaloids. Cancer Cell Int 12: 14, 2012.

6. Ivliev AE, 't Hoen PA and Sergeeva MG: Coexpression network analysis identifies transcriptional modules related to proastrocytic differentiation and sprouty signaling in glioma. Cancer Res 70: 10060-10070, 2010.

7. Rong Y, Belozerov VE, Tucker-Burden C, et al: Epidermal growth factor receptor and PTEN modulate tissue factor expression in glioblastoma through JunD/activator protein-1 transcriptional activity. Cancer Res 69: 2540-2549, 2009.

8. Mazzoleni S, Politi LS, Pala M, et al: Epidermal growth factor receptor expression identifies functionally and molecularly distinct tumor-initiating cells in human glioblastoma multiforme and is required for gliomagenesis. Cancer Res 70: 7500-7513, 2010.

9. Martinez I and Dimaio D: B-Myb, cancer, senescence, and microRNAs. Cancer Res 71: 5370-5373, 2011.

10. Ibrahim AF, Weirauch U, Thomas M, et al: MicroRNA replacement therapy for miR-145 and miR-33a is efficacious in a model of colon carcinoma. Cancer Res 71: 5214-5224, 2011.

11. Farazi TA, Horlings HM, Ten Hoeve JJ, et al: MicroRNA sequence and expression analysis in breast tumors by deep sequencing. Cancer Res 71: 4443-4453, 2011.

12. Liu N, Landreh M, Cao K, et al: The microRNA miR-34 modulates ageing and neurodegeneration in Drosophila. Nature 482: 519-523, 2012.

13. Wu DG, Wang YY, Fan LG, et al: MicroRNA-7 regulates glioblastoma cell invasion via targeting focal adhesion kinase expression. Chin Med J 124: 2616-2621, 2011.

14. Nguyen HT, Dalmasso G, Yan Y, et al: MicroRNA-7 modulates CD98 expression during intestinal epithelial cell differentiation. J Biol Chem 285: 1479-1489, 2010.

15. Reddy SD, Ohshiro K, Rayala SK and Kumar R: MicroRNA-7, a homeobox D10 target, inhibits p21-activated kinase 1 and regulates its functions. Cancer Res 68: 8195-8200, 2008.

16. Webster RJ, Giles KM, Price KJ, et al: Regulation of epidermal growth factor receptor signaling in human cancer cells by microRNA-7. J Biol Chem 284: 5731-5741, 2009.

17. Xiong S, Zheng Y, Jiang P, et al: MicroRNA-7 inhibits the growth of human non-small cell lung cancer A549 cells through targeting BCL-2. Int J Biol Sci 7: 805-814, 2011.

18. Lu J, Getz G, Miska EA, et al: MicroRNA expression profiles classify human cancers. Nature 435: 834-838, 2005.

19. Hu BS, Tan JW, Zhu GH, et al: WWOX induces apoptosis and inhibits proliferation of human hepatoma cell line SMMC-7721. World J Gastroenterol 18: 3020-3026, 2012.

20. Yoo AS, Sun AX, Li L, et al: MicroRNA-mediated conversion of human fibroblasts to neurons. Nature 476: 228-231, 2011.

21. Xiong $\mathrm{F}$, Wu C, Chang J, et al: Genetic variation in an miRNA-1827 binding site in MYCL1 alters susceptibility to small-cell lung cancer. Cancer Res 71: 5175-5181, 2011.

22. Zhou X, Ren Y, Moore L, et al: Downregulation of miR-21 inhibits EGFR pathway and suppresses the growth of human glioblastoma cells independent of PTEN status. Lab Invest 90: 144-155, 2010.

23. Dong H, Luo L, Hong S, et al: Integrated analysis of mutations, miRNA and mRNA expression in glioblastoma. BMC Syst Biol 4: $163,2010$.

24. Huang PH, Miraldi ER, Xu AM, et al: Phosphotyrosine signaling analysis of site-specific mutations on EGFRvIII identifies determinants governing glioblastoma cell growth. Mol Biosyst 6: $1227-1237,2010$.

25. Lal B, Goodwin CR, Sang Y, et al: EGFRvIII and c-Met pathway inhibitors synergize against PTEN-null/EGFRvIII+ glioblastoma xenografts. Mol Cancer Ther 8: 1751-1760, 2009.

26. Reznik TE, Sang Y, Ma Y, et al: Transcription-dependent epidermal growth factor receptor activation by hepatocyte growth factor. Mol Cancer Res 6: 139-150, 2008.

27. Huang PH, Cavenee WK, Furnari FB and White FM: Uncovering therapeutic targets for glioblastoma: a systems biology approach. Cell Cycle 6: 2750-2754, 2007.

28. Chou YT, Lin HH, Lien YC, et al: EGFR promotes lung tumorigenesis by activating miR-7 through a Ras/ERK/Myc pathway that targets the Ets2 transcriptional repressor ERF. Cancer Res 70: $8822-8831,2010$ 
29. Kirubakaran P, Kothapalli R, Singh KhD, et al: In silico studies on marine actinomycetes as potential inhibitors for Glioblastoma multiforme. Bioinformation 6: 100-106, 2011.

30. Kakkar A, Suri V, Jha P, et al: Loss of heterozygosity on chromosome $10 \mathrm{q}$ in glioblastomas, and its association with other genetic alterations and survival in Indian patients. Neurol India 59: 254-261, 2011

31. Halatsch ME, Löw S, Hielscher T, et al: Epidermal growth factor receptor pathway gene expressions and biological response of glioblastoma multiforme cell lines to erlotinib. Anticancer Res 28: 3725-3728, 2008.

32. Motomura K, Natsume A, Kishida Y, et al: Benefits of interferon- $\beta$ and temozolomide combination therapy for newly diagnosed primary glioblastoma with the unmethylated MGMT promoter: a multicenter study. Cancer 117: 1721-1730, 2011.

33. Griffero F, Daga A, Marubbi D, et al: Different response of human glioma tumor-initiating cells to epidermal growth factor receptor kinase inhibitors. J Biol Chem 284: 7138-7148, 2009.

34. Cesana M, Cacchiarelli D, Legnini I, et al: A long noncoding RNA controls muscle differentiation by functioning as a competing endogenous RNA. Cell 147: 358-369, 2011.

35. Patterson EE, Holloway AK, Weng J, et al: MicroRNA profiling of adrenocortical tumors reveals miR-483 as a marker of malignancy. Cancer 117: 1630-1639, 2011.
36. Paroo Z, Ye X, Chen S and Liu Q: Phosphorylation of the human microRNA-generating complex mediates MAPK/Erk signaling. Cell 139: 112-122, 2009.

37. Hide T, Takezaki T, Nakatani Y, et al: Combination of a ptgs2 inhibitor and an epidermal growth factor receptor-signaling inhibitor prevents tumorigenesis of oligodendrocyte lineagederived glioma-initiating cells. Stem Cells 29: 590-599, 2011.

38. Fu S and Kurzrock R: Development of curcumin as an epigenetic agent. Cancer 116: 4670-4676, 2010.

39. Zhu H, Acquaviva J, Ramachandran P, et al: Oncogenic EGFR signaling cooperates with loss of tumor suppressor gene functions in gliomagenesis. Proc Natl Acad Sci USA 106: 2712-2716, 2009.

40. Löw S, Vougioukas VI, Hielscher T, et al: Pathogenetic pathways leading to glioblastoma multiforme: association between gene expressions and resistance to erlotinib. Anticancer Res 28: 3729-3732, 2008.

41. Kefas B, Godlewski J, Comeau L, et al: microRNA-7 inhibits the epidermal growth factor receptor and the Akt pathway and is down-regulated in glioblastoma. Cancer Res 68: 3566-3572, 2008. 